\title{
Nivolumab without brain radiotherapy is insufficient for the treatment of most patients with brain metastases from clear cell renal cell carcinoma
}

\author{
Amit K. Chowdhry, Sara J. Hardy, Michael T. Milano \\ Department of Radiation Oncology, University of Rochester Medical Center, Rochester, NY, USA \\ Correspondence to: Michael T. Milano, MD, PhD. Department of Radiation Oncology, University of Rochester Medical Center, 601 Elmwood Avenue, \\ Box 647, Rochester, NY 14642, USA. Email: Michael_Milano@urmc.rochester.edu. \\ Provenance: This is an invited article commissioned by the Section Editor Dr. Xiao Li (Department of Urology, Jiangsu Cancer Hospital, Jiangsu \\ Institute of Cancer Research, Nanjing Medical University Affiliated Cancer Hospital, Nanjing, China). \\ Comment on: Flippot R, Dalban C, Laguerre B, et al. Safety and Efficacy of Nivolumab in Brain Metastases From Renal Cell Carcinoma: Results of \\ the GETUG-AFU 26 NIVOREN Multicenter Phase II Study. J Clin Oncol 2019;37:2008-16.
}

Submitted Aug 20, 2019. Accepted for publication Aug 30, 2019.

doi: $10.21037 / \mathrm{atm} .2019 .09 .10$

View this article at: http://dx.doi.org/10.21037/atm.2019.09.10

In the article "Safety and Efficacy of Nivolumab in Brain Metastases from Renal Cell Carcinoma: Results of the GETUG-AFU 26 NIVOREN Multicenter Phase II Study" (1), the authors investigated the anti-programmed cell death-1 (PD-1) inhibitor, nivolumab, in patients with brain metastases from clear cell renal cell carcinoma (RCC). All patients developed progression of disease after previously receiving vascular endothelial growth factor-directed therapies. This highly valuable study adds important knowledge to our understanding of the efficacy of immunotherapy in patients with brain metastases from RCC. Most published trials of immunotherapy for RCC have excluded patients with brain metastases. To our knowledge, this is the first prospective study to investigate immunotherapy for brain metastases from RCC.

\section{Methodology and results}

All patients in the NIVOREN study had asymptomatic brain metastases not requiring corticosteroids for symptom control. Two cohorts were enrolled: (I) patients with at least one previously untreated brain metastases ( $\mathrm{n}=39$ ); (II) patients with all brain metastases treated with prior resection and/or radiotherapy $(n=34)$. All patients received intravenous nivolumab every 2 weeks until disease progression. The primary endpoint was intracranial response in cohort A. Four out of 34 patients (12\%) in cohort A had an objective intracranial response. The median intracranial progressionfree survival was 2.7 months in cohort A, with $72 \%$ of patients needing subsequent focal brain therapy (surgery or radiation). The median intracranial progression-free survival in cohort B (those with prior treatment of brain metastases) was 4.8 months. The median extracranial progressionfree survival was 2.8 months in cohort A and 2.6 months in cohort B. The authors concluded that nivolumab has limited efficacy in the treatment of patients with brain metastases from RCC.

\section{Statistical and study design considerations}

The primary limitations of this study from a methodologic standpoint are that there was no randomization and no control group, and the sample size was limited. The study used a Fleming one-stage phase II study-design to determine whether nivolumab is worthy of further study in this subset of patients. The study pre-specified a response probability at which one would deem the drug worthy of further study. The analysis was based upon an estimate of the probability that patients' RCC will have a response to therapy. A unique aspect of this approach is that it employs a one-sided $5 \%$ type I error (false positive rate). While this was appropriate for the study, it is important to note that this is twice the type I error rate of a two-sided test. In this situation, a one-sided test asks the question: is the 
intracranial response rate in cohort A significantly greater than the pre-specified criteria. If a two-sided test were used instead, there would be a $10 \%$ type I error probability. For confirmatory clinical trials, the FDA requires a type I probability of $2.5 \%$ for one-sided tests, and $5 \%$ for a two-sided test. The reason that a one-sided test was used in study was to determine whether the drug is worthy of further study, rather than for efficacy. Therefore, final conclusions of efficacy should be performed using a $2.5 \%$ alpha level if one were to use this to determine efficacy. This is an important consideration when comparing studies with exploratory Phase II designs to conventional phase III designs. Though from a purely methodological standpoint it would be preferable to include patients not treated with nivolumab to fully characterize its effect intracranially relative to placebo or another therapy without known intracranial effect, it would be difficult ethically to justify the use of placebo or another agent without intracranial activity and without local therapy in this patient population. It is also important to note that this study used progressionfree survival and objective response. While appropriate for a phase II study, these are not patient-oriented outcomes. This study reports overall survival, but it is not the primary endpoint. Recent research demonstrates that objective response does not necessarily correlate with overall survival for immunotherapy trials (2). Presumably if the study's results had been more suggestive of treatment effect, overall survival would have been incorporated into further studies. However, brain metastasis control may have a more direct association with patient-oriented outcomes than for metastatic tumor control of disease at other sites, given the high likelihood of symptomatic progression with uncontrolled brain metastases.

The NIVOREN study used RECIST criteria to evaluate response to treatment. One criticism of using RECIST criteria to measure the overall response rate is that it categorizes information about response to treatment, resulting in a loss of information. With RECIST criteria, a $29 \%$ and $31 \%$ decrease in tumor size will be categorized as stable disease and partial response respectively. However, clinically there is no difference between these tumor size changes. Rather than using overall response rate one may use continuous measure of change in tumor size as the outcome, which does not lose information contained in the continuous measurement of tumor sizes (3). Another criticism of the RECIST criteria is that it may not take into account pseudoprogression, which can occur with brain lesions for patients on immunotherapy. Other criteria such as the immunotherapy response assessment for neurooncology (iRANO) have been developed to address the challenges in evaluating intracranial response while on immunotherapy (4).

This study used a regression approach to adjusting the hazard ratio between cohorts $\mathrm{A}$ and $\mathrm{B}$. The investigators did not employ any causal inference methodology to aid in determining whether the local treatment is associated with causal effects. There is an interesting causal question: does prior local treatment of brain metastases improve progression-free survival? While regression methods can be helpful in asking such questions, the employment of modern causal inference methodology such as propensity score matching would add additional robustness which could employ a model that does not rely on the proportional hazards assumption (albeit with a separate set of assumptions regarding the distribution of the propensity scores).

\section{Safety and efficacy of nivolumab on brain metastases from clear cell renal carcinoma}

We agree with the NIVOREN study authors that nivolumab has an acceptable safety profile in this cohort. Though the sample size was small, a significant majority of patients experienced disease progression during the follow-up interval. The overall response rate was $11.8 \%$ [95\% confidence interval (CI): 3.3-27.5\%] in cohort A, which suggests that while the cancer in some patients with intracranial clear cell RCC responds to nivolumab, the vast majority do not. However, it is important to note that the $\mathrm{CI}$ is wide for this estimate.

The most striking cases of patients treated with immunotherapy are those of "exceptional responders," a small subset of patients who experience a dramatic response to therapy. Despite the disappointing response among the study cohort, all $12 \%$ of the patients in cohort A who had an objective intracranial response (those with previously untreated brain metastases) experienced a complete response of their intracranial disease. While studies like this are inadequately powered to answer questions relating to this subset of patients, disparate responses lead to the question to whether we can find assays to determine which patients will potentially have a dramatic response to therapy, allowing better selection of patients with brain metastases from RCC who would benefit from nivolumab.

Another important result was that 6 out of 34 patients $(18 \%)$ had discordant body and brain treatment responses. 
This is consistent with previous work demonstrating discrepancy in targetable mutations in primary tumors, lymph node metastases, and extracranial metastases compared with brain metastases (5). We may not be able to discern which patients will have intracranial response to nivolumab based on analysis of tissue from primary tumor or extracranial metastases, but may have to find means of evaluating the mutation status of the brain metastasis.

\section{The role of local therapy}

One of the most interesting conclusions of the study is that there is, for intracranial progression, an adjusted hazard ratio of 2.04 (95\% CI: $1.08-3.83$ ) between cohort A (the cohort with at least one previously focally untreated brain metastases) and cohort B (the cohort with all previously treated brain metastases). Seventy-two percent of the patients in cohort A received local therapy while on study, and $46 \%$ developed symptomatic progression of their brain metastases. This strongly supports a role for upfront treatment of brain metastases from clear cell RCC. The roles for surgery and radiosurgery for treating brain metastases RCC are supported by decades of research. Randomized data (albeit not specific for RCC) supports that resection affords a survival benefit for patients with a single brain metastasis (specifically surgery and WBRT compared with WBRT alone) (6). Surgery also provides more diagnostic certainty by providing the opportunity for pathologic diagnosis. Stereotactic radiosurgery (SRS), which facilitates the delivery of high doses of radiation in a single or a few fractions, is an option for patients with unresectable brain metastases, or for whom resection may not be indicated. Though RCC is classically thought of as a radioresistant tumor which often does not respond well to conventional radiotherapy (7), SRS has been shown to be efficacious for treating brain metastases from RCC, and is far less likely, compared to whole-brain radiation therapy (WBRT), to cause cognitive decline (8). Though the tumoricidal mechanism for conventionally fractionated therapy is thought to be from mitotic death as a result of DNA damage, the mechanism of action of SRS is currently not fully understood, though is likely a result of vascular injury (9). The high doses of SRS may augment antitumor immunity. This immune-mediated response could potentially lead to an abscopal response (a response in a non-target lesion) which may work synergistically with immune therapy and may increase its effectiveness $(9,10)$. A recent systematic review and meta-analysis suggests that concurrent SRS and immunotherapy may be associated with improved efficacy and safety versus being treated nonconcurrently (11). However, more research is needed to better understand the relationship between immunotherapy and radiotherapy.

\section{Conclusions}

This study demonstrates limited efficacy of nivolumab for the treatment of brain metastases. It is a valuable contribution to the literature on this important question. While this study was not designed to study the effects of local therapy on control of RCC, the results strongly suggest that local control with either radiation or surgery are important treatments in these patients.

\section{Acknowledgments}

None.

\section{Footnote}

Conflicts of Interest: MT Milano: royalties from Wolters Kluwer (UpToDate); the other authors have no conflicts of interest to declare.

Ethical Statement: The authors are accountable for all aspects of the work in ensuring that questions related to the accuracy or integrity of any part of the work are appropriately investigated and resolved.

\section{References}

1. Flippot R, Dalban C, Laguerre B, et al. Safety and Efficacy of Nivolumab in Brain Metastases From Renal Cell Carcinoma: Results of the GETUG-AFU 26 NIVOREN Multicenter Phase II Study. J Clin Oncol 2019;37:2008-16.

2. Mushti SL, Mulkey F, Sridhara R. Evaluation of overall response rate and progression-free survival as potential surrogate endpoints for overall survival in immunotherapy trials. Clin Cancer Res 2018;24:2268-75.

3. George SL, Wang X, Pang H. Cancer Clinical Trials: Current and Controversial Issues in Design and Analysis. CRC Press, 2016.

4. Okada H, Weller M, Huang R, et al. Immunotherapy response assessment in neuro-oncology: a report of the RANO working group. Lancet Oncol 2015;16:e534-42. 


\section{Page 4 of 4}

5. Brastianos PK, Carter SL, Santagata S, et al. Genomic Characterization of Brain Metastases Reveals Branched Evolution and Potential Therapeutic Targets. Cancer Discov 2015;5:1164-77.

6. Patchell RA, Tibbs PA, Walsh JW, et al. A randomized trial of surgery in the treatment of single metastases to the brain. N Engl J Med 1990;322:494-500.

7. Wrónski M, Maor MH, Davis BJ, et al. External radiation of brain metastases from renal carcinoma: a retrospective study of 119 patients from the M. D. Anderson Cancer Center. Int J Radiat Oncol Biol Phys 1997;37:753-9.

8. Chang EL, Wefel JS, Hess KR, et al. Neurocognition in patients with brain metastases treated with radiosurgery or radiosurgery plus whole-brain irradiation: a randomised

Cite this article as: Chowdhry AK, Hardy SJ, Milano MT. Nivolumab without brain radiotherapy is insufficient for the treatment of most patients with brain metastases from clear cell renal cell carcinoma. Ann Transl Med 2019;7(Suppl 8):S366. doi: 10.21037/atm.2019.09.10

\section{Chowdhry et al. Nivolumab alone for RCC brain metastases}

controlled trial. Lancet Oncol 2009;10:1037-44.

9. Sharabi AB, Lim M, DeWeese TL, et al. Radiation and checkpoint blockade immunotherapy: radiosensitisation and potential mechanisms of synergy. Lancet Oncol 2015;16:e498-509.

10. Chakraborty M, Abrams SI, Coleman CN, et al. External beam radiation of tumors alters phenotype of tumor cells to render them susceptible to vaccine-mediated T-cell killing. Cancer Res 2004;64:4328-37.

11. Lehrer EJ, Peterson J, Brown PD, et al. Treatment of brain metastases with stereotactic radiosurgery and immune checkpoint inhibitors: An international meta-analysis of individual patient data. Radiother Oncol 2019;130:104-12. 